\title{
Estudio comparativo entre la atención del alumbramiento activo modificado y el alumbramiento activo
}

\author{
Oswaldo J. Cárdenas $\mathrm{H}$. \\ Facultad de Ciencias Médicas, Universidad de Cuenca, Cuenca \\ Autor para correspondencia: oswaldo.cardenas@ucuenca.edu.ec \\ Fecha de recepción: 4 de enero de 2012 - Fecha de aceptación: 27 de julio de 2012
}

\section{RESUMEN}

Se inició un proyecto de investigación para comparar el método de atención del alumbramiento activo modificado con el método activo durante el tercer periodo del parto. En el método de atención del alumbramiento activo modificado se utilizó un signo directo para verificar el descenso placentario, el examen vaginal, en contraste con el método de los indicadores indirectos prescritos por el Ministerio de Salud Pública del Ecuador. Una muestra aleatorizada de 300 mujeres en labor de parto fueron atendidas en cada grupo. La investigación fue realizada en el Hospital Vicente Corral Moscoso de Cuenca, Ecuador, y fueron monitorizadas las siguientes variables durante el tercer periodo de atención del parto para evaluar el comportamiento de ambos métodos de atención: duración del tercer periodo del parto, pérdida de sangre, frecuencia de la retención parcial y total placentaria, volumen de la pérdida de sangre durante la primera hora posterior al tercer periodo del parto. Los resultados revelaron que el promedio de duración del tercer periodo del parto en el alumbramiento activo modificado fue 4,35 $\pm 1,36$ versus 4,22 $\pm 1,60$ minutos en el alumbramiento activo con un valor de $p$ igual a 0,297 , que indica que la duración del tercer periodo del parto en los dos tipos de atención fue similar. La media de la pérdida de sangre en el método activo modificado fue $193 \pm 66,97$ versus $182,97 \pm 54,62$ cc para la atención del alumbramiento activo con un valor de p igual a 0,045. La investigación comparativa reveló que las dos formas de atención durante el tercer periodo del parto presentaron resultados iguales, con beneficio para la forma de atención activa.

Palabras clave: Tercer periodo del parto, alumbramiento activo modificado, alumbramiento activo.

\begin{abstract}
A research project was initiated to compare the modified-active versus active attention method during the third labor phase in childbirth. In the modified-active attention method indirect indicators are used to verify the placental descent, in contrast to the vaginal examination in the active attention approach, a requirement prescribed by the Ecuadorian Ministry of Health. A random sample of 300 women in labor was subjected to each attention approach. The research was conducted at the Vicente Corral Moscoso Hospital of Cuenca, Ecuador, and the following variables were monitored during the third labor phase to assess the performance of both attention methods: duration of the third stage of labor, blood loss, the frequency of total and partial placental retention, and the volume of blood loss during the first hour posterior the third stage of labor. Results revealed that the average length of the modified-active third stage of labor was 4,35 $\pm 1,36$ and the active third stage of labor 4,22 $\pm 1,60$ minutes with a $p$ value of 0,297 , indicating that the duration of the third stage of the two types of attention methods are similar. The average blood loss of the modified-active third stage of labor was $193,01 \pm 66,97$ versus $182,97 \pm 54,62$ cc for the active attention approach with a $p$ value of 0,045 . The comparative research revealed that the two forms of attention during the third stage of labor perform equally well, with a slight preference for the active form of attention.
\end{abstract}

Keywords: Third stage of labor, modified-active procedure, active procedure. 


\section{INTRODUCCIÓN}

El 99\% de las abundantes hemorragias posparto se producen en países en desarrollo y constituyen una de las tres principales causas de morbilidad y mortalidad materna ecuatoriana y mundial (Organización Mundial de la Salud, 2001; Schwarcz y col., 2005; Pérez Sánchez y Donoso Siña, 2011). La comunidad científica obstétrica nacional e internacional ha tratado de disminuir esta morbilidad y mortalidad (Carrera y col., 2006; Botero y col., 2008), reemplazando la atención del alumbramiento expectante con el alumbramiento activo, actualmente norma nacional del Ministerio de Salud Pública (MSP) Ecuatoriano, que utiliza ocitocina después del nacimiento del feto, tracción controlada del cordón umbilical y masaje uterino. La tracción controlada del cordón umbilical se realiza luego de observar los signos indirectos del desprendimiento placentario y se ejecuta reiteradamente hasta conseguir la expulsión placentaria.

El manejo del alumbramiento activo, también denominado dirigido se ha utilizado en muchos tratados de obstetricia modernos para referirse a la administración de fármacos con potencialidad de estimular la contractibilidad uterina, luego de la extracción del feto, seguida de maniobras para conseguir un aceleramiento de la expulsión placentaria (Rogers y col., 1998; Prendville y col., 2000; McDonald y col., 2002). Existen diversas publicaciones que describen el uso de ocitocina más tracción controlada del cordón umbilical para la atención del período placentario (Cárdenas, 2003; Cifuentes, 2009). El objetivo general del tratamiento del alumbramiento activo, según Prendville (1998), es la reducción de los siguientes riesgos: hemorragia posparto de más de 500 mililitros [riesgo relativo (RR) de 0,38, intervalo de confianza (IC95\%) de 0,32 a 0,46]; y prolongación del tercer estadio del trabajo de parto (diferencia promedio balanceada 9,77 minutos, IC95\% de 9,53 a 10).

En un estudio de Cárdenas y col. (2003) se utilizó el tacto vaginal, previo a la tracción controlada del cordón umbilical, comparando el alumbramiento expectante con el alumbramiento activo, con una muestra de 200 embarazadas, 100 en cada grupo, sin la aplicación de ocitocina en los dos grupos. Se obtuvo un RR de 0,56, IC95\% de 0,43 a 0,68 para la duración del alumbramiento activo para tiempos mayores a 5 minutos, y para cantidades de hemorragia mayores a $200 \mathrm{cc}$ un RR de 0,54, IC95\% de 0,40 a 0,72. Cifuentes (2009) recomienda el manejo activo del alumbramiento combinando el uso de ocitócicos y tracción controlada del cordón y esperar 30 minutos para cualquier intervención en el tercer periodo. Quintero y col. (1999) encontraron que la tracción controlada del cordón umbilical, efectuada sin observar los signos objetivos de desprendimiento placentario, alarga el tiempo de duración del alumbramiento y aumenta la cantidad de hemorragia. El MSP del Ecuador implementó, en sus capítulos normativos, el alumbramiento activo, dentro del Plan Nacional de Reducción Acelerada de la Muerte Materna y Neonatal, como política pública prioritaria para el sector de salud, en su norma y protocolo materno (Ministerio de Salud Pública, 2008). Estimamos que la comprobación manual del descenso placentario, mediante el tacto del tercio inferior de la vagina, realizado a los 3 minutos de la expulsión del feto y la verificación manual de que la placenta se halla por debajo del borde cervical uterino, constituye el mejor método obstétrico para comprobar el desprendimiento y comenzar la tracción del cordón umbilical, siendo el indicador más calificado para iniciar las maniobras de la expulsión de la placenta.

Un procedimiento alternativo al alumbramiento activo es el alumbramiento activo modificado que recomienda el tacto del tercio inferior vaginal, luego del nacimiento del feto y de la administración de ocitocina, para comprobar digitalmente que la placenta se halla por debajo del cuello uterino e iniciar la tracción controlada del cordón umbilical, para extraer la placenta de inmediato y con seguridad, con la finalidad de acortar significativamente el tiempo de duración del alumbramiento y disminuir la pérdida de sangre. En la práctica, no hay ningún protocolo establecido científico y estadísticamente sustentado que nos permita adoptar un manejo activo modificado que inicie tempranamente las maniobras de expulsión placentaria, comprobando la expulsión placentaria manualmente y directamente, mediante el tacto vaginal. Por esta razón, los autores iniciaron una investigación experimental clínica aleatorizada, para comparar si el método de atención del alumbramiento activo modificado propiciará menor tiempo de duración del alumbramiento y menor pérdida de sangre durante el alumbramiento si lo comparamos con el alumbramiento activo clásico. Indicadores adicionales en el estudio experimental fueron la frecuencia de la retención parcial o total 
placentaria y la cantidad de la pérdida de sangre durante la primera hora posalumbramiento en los dos tipos de atención del alumbramiento.

\section{MATERIALES Y MÉTODOS}

Se realizó una investigación clínica aleatorizada, experimental y comparativa, entre la atención del alumbramiento activo modificado (utilizando la verificación del desprendimiento placentario mediante tacto del tercio inferior de la vagina) y la atención del alumbramiento activo, que constituye la norma del MSP de Ecuador. El universo de estudio lo conformaron las embarazadas que acudieron en labor de parto, para ser atendidas en el Hospital Vicente Corral Moscoso, desde el 1 de noviembre del 2009 al 30 de septiembre del 2010.

Para el cálculo de la muestra se consideraron los resultados de una investigación anterior, realizada por el autor, donde se obtuvieron los siguientes resultados: media del tiempo de duración del alumbramiento activo 5,86 minutos; se espera reducir a 4,45 minutos, es decir disminuir del 50 al $38 \%$. Media para la cantidad de hemorragia del alumbramiento activo $169 \mathrm{cc}$; se espera reducir a 128 cc, es decir del 50 al 38\%. Para el cálculo de la muestra se utilizó el programa EpiInfo ${ }^{\mathrm{TM}}$ de la Organización Mundial de la Salud (PROGRAMAS / STATCALC / Muestras: tamaño y poder): nivel de confianza (IC) 95\%; probabilidad de que si las dos poblaciones difieren, las dos muestras expresen una diferencia "significativa" poder (1- $\beta$ ) de $80 \%$; razón de no expuestos/número de expuestos, 1:1; prevalencia esperada del factor en grupo no expuestas $50 \%$; prevalencia del factor en expuestas $38 \%$. Se obtuvo una muestra de 284 para las no expuestas y para las expuestas. Para dar una mayor significación estadística, y estudiar en los dos grupos de embarazadas los dos puntos finales, se utilizó una muestra de 300 embarazadas para el alumbramiento activo modificado y para el alumbramiento activo, que supera el valor obtenido en el cálculo inicial de la muestra.

Se conformaron dos grupos asignando las 600 embarazadas, sujetos de la investigación, en dos grupos de 300 (150 primigestas y 150 multíparas en cada grupo), mediante un procedimiento randomizado utilizando una tabla de números aleatorios para bloques de 20 números, para primigestas y multíparas, luego de haber cumplido con los criterios de inclusión y no tener criterios de exclusión. Previamente se registró la firma, de la seleccionada, en el consentimiento informado. El proceso de inclusión de las embarazadas en la investigación fue continuo, y se realizó en mujeres embarazadas, consideradas como normales para la atención del parto, que acudieron en labor de parto, a término, al Centro Obstétrico del Hospital Regional Vicente Corral Moscoso. No se incluyeron a las embarazadas que presentaron patología general; antecedente de cesárea; necesidad de conducción o inducción del parto; complicaciones maternas, fetales, materno-fetales o clínicas durante el primer periodo y/o segundo periodo del parto; embarazadas que requirieron tratamientos adicionales; partos instrumentales, manifestaron su deseo de no ingresar a la investigación o existió una gran prolongación del primero y segundo periodo del parto, de acuerdo al partograma del Centro Latinoamericano de Perinatología (CLAP).

Las variables del estudio fueron: variables de control, independientes y dependientes. La variables de control consistieron en edad materna, antecedente de gestaciones, antecedente de partos, talla de la embarazada, peso de la embarazada, índice de masa corporal, talla del recién nacido, peso del recién nacido. Los dos métodos, el alumbramiento activo modificado y el alumbramiento activo representaron las variables independientes. Las variables dependientes, para determinar cuál de los métodos de atención es el mejor fueron: el tiempo de duración del alumbramiento, la cantidad de hemorragia, las complicaciones como la retención de membranas, lóbulos placentarios, expulsión total de la placenta, y la hemorragia durante la primera hora del pos alumbramiento.

Para mensurar el tiempo de duración del alumbramiento, en minutos, se utilizó un reloj electrónico digital de alta precisión. La medición de la cantidad de sangre, recolectada durante la atención del alumbramiento, se efectuó en un recipiente de laboratorio. La medición del peso y de la talla de la embarazada se realizó en la balanza-tallímetro Health Meter Professional. Las observaciones de los investigadores, el cumplimiento de la metodología de la atención de los dos 
procedimientos de atención del alumbramiento, la medición de la cantidad de sangre, la mensuración del tiempo de duración del alumbramiento, así como la evaluación de la integridad de la placenta, fueron confrontadas por los médicos residentes colaboradores de la investigación, previo al inicio de la investigación, para que no existan diferencias en la atención a la embarazada y en la obtención de los datos. En el estudio se aplicaron las normas de bioética internacionales. Además, se archivó el original del consentimiento informado, firmado por la embarazada, en el expediente de la investigación.

Para el registro de los resultados se utilizó un formulario codificado elaborado en el programa de investigación EpiInfo ${ }^{\mathrm{TM}}$. Para el análisis de los datos se utilizó el programa SPSS versión 15. Los resultados primarios en los dos grupos de atención del alumbramiento y las complicaciones se expresaron, para las variables cuantitativas continuas (variables primarias: cantidad de hemorragia y tiempo de duración del alumbramiento) mediante diferencias de medias entre los dos grupos y el valor de p para conocer la significación de las diferencias. Para la presencia o ausencia de complicaciones, así como para hemorragias mayores a $200 \mathrm{cc}$, mayores a $300 \mathrm{cc}$ y tiempos de duración del alumbramiento de 3 minutos, se expresaron mediante RR, IC, reducción del riesgo relativo (RRR), reducción del riesgo absoluto (RRA) y número necesario a tratar (NNT).

\section{INTERVENCIÓN PROPUESTA}

En los dos grupos de atención, el alumbramiento activo modificado y activo, la vigilancia y atención del parto para el primero y segundo periodo se realizó utilizando iguales prácticas obstétricas, beneficiosas probadas y aceptadas por la medicina basada en evidencias. Obtenida la expulsión fetal se diferenció, en cada uno de los dos grupos, la atención del tercer periodo del parto. La diferencia en los dos tipos de atención del alumbramiento consistió en la identificación y obtención de los indicadores obstétricos que sirven para iniciar la tracción del cordón umbilical. Más detalle de cada método de atención durante el tercer periodo se presenta posteriormente. Se efectuó la atención del cuarto periodo del parto de acuerdo a las normas del MSP en los dos grupos. Se vigiló clínicamente la hemorragia durante la primera hora del posparto, contando el número de toallas utilizadas, que se registró en el formulario de la investigación. De cada grupo se registraron las complicaciones del alumbramiento en lo relacionado con la retención total o parcial de la placenta o membranas y la cantidad abundante de hemorragia.

\subsection{Procedimiento de atención del alumbramiento activo modificado}

Se registró en horas y minutos el nacimiento del feto y de inmediato se procedió a pinzar doblemente y a cortar el cordón cambiando el recipiente de recolección de sangre. El pinzamiento se realizó a nivel del introito vaginal. Se verificó el desprendimiento placentario con el tacto, solo del tercio inferior de la vagina, comprobando que la placenta se halla por debajo del cuello uterino y se procedió a la atención del alumbramiento iniciando la tracción del cordón umbilical, sin considerar los indicadores de la norma del MSP. Luego de la salida del feto, se palpó el abdomen materno para descartar la presencia de otro u otros fetos.

\section{Administración de un útero tónico}

Se administró oxitocina 10 unidades, por vía intramuscular (IM) y se registró en la historia clínica del MSP.

Tracción controlada del cordón umbilical

Se acercó la pinza, que está en el cordón umbilical, hasta el periné. Se sostuvo el cordón pinzado y se mantuvo tensión leve continua en el cordón umbilical con una mano y con la otra se realizó un tacto del tercio vaginal inferior, a los 3 minutos del nacimiento de feto. Para realizar el tacto vaginal no se esperó una contracción fuerte del útero, ni el descenso de la pinza aplicada en el cordón umbilical, como recomienda la norma de atención del MSP. Si se palpó la placenta por debajo del cuello uterino, 
se interpretó como un indicador de que la placenta se ha desprendido y se hallaba parcialmente fuera del útero. De inmediato se efectuó su extracción, mediante tracción del cordón umbilical. Se colocó la otra mano apenas por encima del pubis de la mujer, se estabilizó el útero aplicando siempre contracción durante la tracción controlada del cordón umbilical, para evitar una inversión uterina. Se mantuvo tensión leve del cordón umbilical hasta que se expulsó totalmente la placenta a través de la vagina. Si la placenta no se palpó por debajo del cuello uterino se esperó 2 minutos y nuevamente se efectuó un tacto vaginal. Si la placenta estaba palpable se realizó la tracción controlada, continua, suave hasta conseguir la expulsión placentaria. Si en el segundo tacto no se palpó la placenta se procedió a realizarlo cada 2 minutos hasta verificar que la placenta se encontraba por debajo del cuello uterino e iniciar la tracción de la placenta con contratracción. Al ser expulsada la placenta, se sostuvo ésta con las dos manos hasta que las membranas se expulsaron. Se verificó y examinó que la placenta y las membranas se encuentren completas. Si se realizó episiorrafia se suturaron los tejidos vaginales o perineales. Previamente se cambiaron los guantes que sirvieron para la atención del periodo expulsivo y del alumbramiento, con el objetivo de no predisponer con el tacto las infecciones vaginales-perineales. Se midió y registró en este momento el tiempo de duración del alumbramiento y la cantidad de sangre recolectada.

\section{Masaje uterino}

Para efectuar el masaje uterino se aplicaron las normas de Atención del Alumbramiento del MSP, en los dos grupos de atención. Se masajeó de inmediato el fondo uterino a través del abdomen de la mujer hasta conseguir que el útero se mantenga contraído. Se repitió el masaje uterino cada 15 minutos durante las 2 primeras horas, luego se aseguró que el útero no se relaje. Se registraron los datos en la historia clínica materno perinatal. En el caso de retención placentaria por más de 30 minutos, con o sin sangrado, se debió realizar la extracción manual de la placenta y/o la revisión de la cavidad bajo anestesia general o sedación profunda. No fue necesario realizarlo porque no existieron retenciones placentarias.

\subsection{Procedimiento de atención para el alumbramiento activo (norma del MSP)}

Se registró en horas y minutos el nacimiento del feto y de inmediato se procedió a pinzar doblemente y a cortar el cordón cambiando el recipiente de recolección de sangre. El pinzamiento se realizó a nivel del introito vaginal. No se comprobó el desprendimiento placentario con el tacto vaginal y se procedió a la atención del alumbramiento activo de acuerdo a la norma del MSP. Luego de la salida del feto, se palpó el abdomen materno para descartar la presencia de otro u otros fetos.

\section{Administración de un útero tónico}

Se administró oxitocina 10 unidades, por vía intramuscular IM y se registró en la historia clínica del MSP.

Tracción controlada del cordón umbilical

Se acercó la pinza que está en el cordón hasta el periné. Se sostuvo el cordón pinzado y el extremo de la pinza con una mano. Se colocó la otra mano apenas por encima del pubis de la mujer, se estabilizó el útero aplicando contratracción. Se mantuvo tensión leve del cordón umbilical y se esperó una contracción fuerte del útero (2-3 minutos). Cuando el útero se contrajo o el cordón se alargó, se haló del cordón con mucha delicadeza para extraer la placenta. Con la otra mano se continuó con contratracción sobre el útero. Si la placenta no descendió después de 30 a 40 segundos de tracción controlada del cordón (es decir no existió ningún signo de separación placentaria), no se continuó halando el cordón. Se sostuvo con delicadeza el cordón umbilical y se esperó hasta que el útero nuevamente esté bien contraído. Con la contratracción siguiente se repitió la tracción controlada del cordón umbilical, manteniendo la contratracción para evitar la inversión uterina. Al ser expulsada la placenta, se sostuvo con las dos manos y se le giró con delicadeza hasta que las membranas queden torcidas y se expulsen. Se verificó y examinó que la placenta y las membranas se expulsaron completamente. Si se realizó episiorrafia se suturaron los tejidos vaginales o perineales. Se utilizaron los mismos guantes que sirvieron para la atención del periodo expulsivo y el alumbramiento, como es costumbre internacional en toda atención del parto. 
Masaje uterino

Se masajeó de inmediato el fondo uterino a través del abdomen de la mujer hasta conseguir que el útero se mantenga contraído. Se repitió el masaje uterino cada 15 minutos durante las 2 primeras horas, luego se aseguró que el útero no se relaje. Se registraron los datos en la historia clínica materno perinatal. En el caso de retención placentaria por más de 30 minutos, con o sin sangrado, se debió realizar la extracción manual de la placenta y/o la revisión de la cavidad bajo anestesia general o sedación profunda. No fue necesario realizarlo porque no existieron retenciones placentarias.

\section{RESULTADOS Y DISCUSIÓN}

Todos los valores de p para las 8 variables de control fueron mayores a 0,05 (Tabla 1), lo que expresa que estas variables, en los dos grupos son estadísticamente iguales, como resultado de una excelente aleatorización, lo que otorga a la investigación la validez interna indispensable en todo estudio experimental, es decir, que el tratamiento experimental, -variables independientes-, y no otros factores son la causa de las diferencias encontradas.

Tabla 1. Características basales de las variables de control de los dos grupos de atención del alumbramiento en 600 embarazadas según los dos grupos de estudio (Hospital Vicente Corral Moscoso, Cuenca, Ecuador, 2010).

\begin{tabular}{lccc}
\hline \multicolumn{1}{c}{ Variables } & $\begin{array}{c}\text { Alumbramiento activo } \\
\text { modificado }\end{array}$ & Alumbramiento activo & $\mathrm{p}$ \\
\hline \multicolumn{1}{c}{ Edad materna } & $23,28 \pm 5,90$ & $23,14 \pm 5,59$ & 0,777 \\
Antecedentes de gestaciones & $2,00 \pm 1,50$ & $2,00 \pm 1,13$ & 0,225 \\
Antecedentes de partos & $2,00 \pm 1,36$ & $2,00 \pm 0,956$ & 0,243 \\
Talla de la embarazada & $151,82 \pm 6,20$ & $152,04 \pm 5,83$ & 0,645 \\
Peso de la embarazada & $142,22 \pm 19,67$ & $141,52 \pm 18,71$ & 0,655 \\
Índice de masa corporal & $28,04 \pm 3,49$ & $27,83 \pm 3,36$ & 0,453 \\
Talla del recién nacido & $48,74 \pm 2,34$ & $48,95 \pm 1,78$ & 0,238 \\
Peso del recién nacido & $6,51 \pm 0,74$ & $6,51 \pm 0,73$ & 0,933 \\
\hline
\end{tabular}

La media del tiempo de duración del alumbramiento en minutos, fue ligeramente mayor (13 segundos) para el alumbramiento activo modificado si lo comparamos con la media del alumbramiento activo. El valor de $\mathrm{p}=0,297$ para el tiempo de duración del alumbramiento no expresa significación estadística, lo que permite deducir que las dos formas de atención del alumbramiento ofrecen resultados estadísticamente similares.

Evaluando el tiempo de duración del alumbramiento, mayor a 3 minutos y de 3 o menor, en los dos tipos de atención del alumbramiento, se encontró un RR de 1,21, mayor a uno, que indica que en el alumbramiento activo modificado se presentaron más embarazadas con tiempos de duración del alumbramiento superior a 3 minutos. El valor de p $(0,004)$ y el IC95\% $(1,06$ a 1,37$)$ expresan significación estadística.

La media de la cantidad de hemorragia en cc, fue ligeramente mayor (una diferencia de 10,04 cc) en el alumbramiento activo modificado. El valor de p $(0,045)$ para la cantidad de hemorragia del alumbramiento expresa significación estadística, lo que permite deducir que las dos formas de atención del alumbramiento ofrecen resultados diferentes con ventaja para el alumbramiento activo. Analizando la pérdida de sangre, entre el número de embarazadas, menor a $200 \mathrm{cc}$, entre el alumbramiento activo modificado y el alumbramiento activo se encontraron 25 embarazadas más en el alumbramiento activo que en el alumbramiento activo modificado, con un valor de p $(0,009)$ significativo lo que permite inferir que el alumbramiento activo modificado produce una menor 
pérdida de sangre, en el rango menor a $200 \mathrm{cc}$, con significación estadística, como lo demuestran los valores del RR $(0,90)$ y el IC95\% $(0,83$ a 0,98$)$.

Tabla 2. Tiempo de duración del alumbramiento en minutos y tiempo de duración del alumbramiento mayor a 3 minutos y de 3 o menos minutos en los dos tipos de atención del alumbramiento en 600 embarazadas (Hospital Vicente Corral Moscoso, Cuenca, Ecuador, 2010).

\begin{tabular}{ccc}
\hline $\begin{array}{c}\text { Tiempo de duración del alumbramiento, } \\
\text { en minutos, en los dos tipos de atención } \\
\text { del alumbramiento }\end{array}$ & $\begin{array}{c}\text { Alumbramiento activo } \\
\text { modificado }\end{array}$ & Alumbramiento activo \\
\hline Media y desvío estándar & $4,35 \pm 1,36$ & $4,22 \pm 1,60$ \\
Mínimo tiempo & 2 & 2 \\
Máximo tiempo & 10 & 15 \\
Mediana & 5 & 4 \\
Tiempo (minutos) $>3$ & 199 & 165 \\
Tiempo (minutos) $\leq 3$ & 101 & 135 \\
\hline
\end{tabular}

Tabla 3. Pérdida de sangre del alumbramiento, en cc, y cantidad de pérdida de sangre del alumbramiento menor a $200 \mathrm{cc}$ e igual y mayor a $200 \mathrm{cc}$, en los dos tipos de atención del alumbramiento en 600 embarazadas (Hospital Vicente Corral Moscoso, Cuenca, Ecuador, 2010).

\begin{tabular}{ccc}
\hline $\begin{array}{c}\text { Cantidad de hemorragia en los dos tipos } \\
\text { de atención del alumbramiento }\end{array}$ & $\begin{array}{c}\text { Alumbramiento activo } \\
\text { modificado }\end{array}$ & Alumbramiento activo \\
\hline Desvío estándar & $193,01 \pm 66,97$ & $182,97 \pm 54,62$ \\
Mínima cantidad & 20 & 50 \\
Máxima cantidad & 600 & 400 \\
Mediana & 200 & 200 \\
Cantidad $<200$ cc & 229 & 254 \\
Cantidad $\geq 200$ cc & 71 & 46 \\
\hline
\end{tabular}

Tabla 4. Duración del alumbramiento, en minutos y cantidad de hemorragia en centímetros cúbicos en relación con la paridad y en los dos tipos de atención del alumbramiento en 600 embarazadas (Hospital Vicente Corral Moscoso, Cuenca, Ecuador, 2010).

\begin{tabular}{cccc}
\hline \multirow{2}{*}{ Paridad } & \multicolumn{2}{c}{ Duración en minutos } & \multirow{2}{*}{$\mathrm{p}$} \\
\cline { 2 - 3 } & $\begin{array}{c}\text { Alumbramiento activo } \\
\text { modificado }\end{array}$ & Alumbramiento activo & \\
\hline Primigestas & $4,36 \pm 1,24$ & $4,33 \pm 1,50$ & 0,835 \\
Multíparas & $4,34 \pm 1,46$ & $4,12 \pm 1,69$ & 0,229 \\
\hline \multirow{4}{*}{ Paridad } & \multicolumn{2}{c}{ Cantidad de hemorragia en cc } & $\mathrm{p}$ \\
& Alumbramiento activo & Alumbramiento activo & \\
\hline Primigestas & modificado & $191,87 \pm 50,86$ & 0,092 \\
Multíparas & $204,13 \pm 72,98$ & $174,07 \pm 56,92$ & 0,242 \\
\hline
\end{tabular}

La media de la duración del alumbramiento en minutos, en primigestas fue mayor en 3 centésimas de minuto, para el alumbramiento activo modificado, con un valor de $\mathrm{p}(0,835)$. La diferencia de las medias de duración del alumbramiento en minutos entre el alumbramiento activo modificado y el alumbramiento activo, en multíparas, fue superior en 22 centésimas de minuto, para 
el alumbramiento activo modificado, con un valor de $\mathrm{p}(0,242)$; que indica que las dos formas de atención del alumbramiento producen resultados estadísticamente iguales.

La media de la cantidad de hemorragia, en centímetros, en primigestas, fue mayor en 12,26 cc para el alumbramiento activo modificado, con un valor de $\mathrm{p}(0,092)$; no significativo si lo comparamos con el alumbramiento activo. La media de la cantidad de hemorragia en multíparas fue mayor en 7,81 cc para el alumbramiento activo modificado, con un valor de $\mathrm{p}(0,242)$, no significativo.

Tabla 5. Retención de restos placentarios y de membranas en 600 embarazadas, según los grupos de atención del alumbramiento (Hospital Vicente Corral Moscoso, Cuenca, Ecuador, 2010).

\begin{tabular}{cccc}
\hline $\begin{array}{c}\text { Retención de restos } \\
\text { placentarios }\end{array}$ & $\begin{array}{c}\text { Alumbramiento activo } \\
\text { modificado }\end{array}$ & Alumbramiento activo & $\mathrm{p}$ \\
\hline $\mathrm{Si}$ & 1 & 0 & 0,317 \\
$\mathrm{No}$ & 299 & 300 & \\
Total & 300 & 300 & $\mathrm{p}$ \\
\hline Retención de & Alumbramiento activo & Alumbramiento activo & 0,317 \\
membranas & modificado & 1 & \\
$\mathrm{Si}$ & 0 & 299 & \\
$\mathrm{No}$ & 300 & 300 & \\
Total & 300 & & \\
\hline
\end{tabular}

Existió una sola retención de restos placentarios en la atención del alumbramiento activo modificado, que se solucionó extrayéndolos digitalmente de la vagina. Solo una madre que presentó retención de membranas en el canal vaginal correspondió a la atención del alumbramiento activo, se extrajo digitalmente de la vagina.

Tabla 6. Número de tactos en embarazadas durante el alumbramiento activo modificado (Hospital Vicente Corral Moscoso, Cuenca, Ecuador, 2010).

\begin{tabular}{cccc}
\hline \multirow{2}{*}{ Número de tactos } & \multicolumn{2}{c}{ Alumbramiento activo modificado } & \\
\cline { 2 - 3 } & Número & Porcentaje & \\
\hline 2 & 229 & 76,3 & \\
\hline 2 & 71 & 23,7 & $\mathrm{p}$ \\
\hline Número de tactos & Primigestas & Multíparas & 0,825 \\
\hline Promedio & $1,75 \pm 0,723$ & $1,77 \pm 0,837$ & $(0,781-1,76)$ \\
Mediana & 2 & 2 & \\
\hline
\end{tabular}

El número de tactos vaginales en 300 embarazadas con alumbramiento activo modificado, para verificar el desprendimiento placentario, realizados solamente en el alumbramiento activo modificado, demostró que el número de 2 tactos, correspondió al mayor número de madres 229 . Si lo comparamos con 3 o más tactos, 71 madres. Se obtuvo (IC95\% de 0,781 a 1,76). Las primigestas presentaron una media menor para el número de tactos, aunque, con un valor de $\mathrm{p}(0,825)$ que expresa que no existe significación estadística. En la atención del alumbramiento activo no se realizaron tactos vaginales, ya que este procedimiento se aplicó solo en la atención del alumbramiento activo modificado, de acuerdo con el diseño de la investigación experimental.

La media para el número de toallas aplicadas en los genitales maternos para recibir los loquios fue ligeramente mayor en el alumbramiento activo modificado 1,54 con desvío estándar de 0,525, si lo comparamos con el número de toallas utilizadas en el alumbramiento activo 1,43 con desvío estándar de 0,535, con significación estadística $(\mathrm{p}=0,009)$. 
En la presente discusión de la investigación no es posible realizar un estudio comparativo de los presentes resultados con otras investigaciones ya que no existen publicaciones sobre esta nueva modalidad de atención del alumbramiento, en la que se utilice el tacto vaginal para verificar el desprendimiento y descenso placentario, método inédito, único en todo el ámbito obstétrico nacional y mundial. Por lo expuesto se realiza en este capítulo un análisis comparativo entre los resultados del alumbramiento activo modificado, el alumbramiento activo y las recomendaciones mundiales sobre la atención del alumbramiento.

Las normas internacionales estiman que en la duración del alumbramiento, debe esperarse, si no se obtiene la salida de la placenta, hasta 30 minutos luego del nacimiento del feto, debiendo de inmediato, realizarse la extracción manual de la placenta. Los presentes resultados determinan que la duración media del alumbramiento activo modificado fue de 4,35 $\pm 1,36$ minutos y del alumbramiento activo de 4,22 \pm 1,60 minutos, es decir, los tiempos medios de duración no sobrepasan los 5 minutos, constituyendo un error grave esperar la eliminación de la placenta hasta por 30 minutos, lapso en el cual se pueden producir complicaciones graves, siendo la más severa el incremento alarmante y peligroso de la cantidad de hemorragia.

Las recomendaciones internacionales consideran que la media de la cantidad de la pérdida de sangre durante el parto sin complicaciones hemorrágicas, es de $500 \mathrm{cc}$. Los resultados de la investigación determinan que la media de pérdida de sangre en el alumbramiento activo modificado fue de 193,01 $\pm 66,97 \mathrm{cc}$ y en el alumbramiento activo de 182,97 $\pm 54,62 \mathrm{cc}$. La media de la pérdida de sangre, en la presente investigación, no supera los $200 \mathrm{cc}$, menor a la media internacional de 500 cc.

Existió una sola retención de restos placentarios en la vagina en la atención del alumbramiento activo modificado y una sola retención de membranas en el canal vaginal en la atención del alumbramiento activo, que se retiraron digitalmente de la vagina.

Sobre el número de tactos vaginales, para verificar el desprendimiento placentario, realizado solamente en el alumbramiento activo modificado, se encontró que para 2 tactos correspondió el mayor número de madres 229, aunque sin significación estadística ( $\mathrm{p}=0,825$; IC95\% de 0,78 a 1,76). La media para el número de toallas aplicadas en los genitales maternos para recibir los loquios fue ligeramente mayor en el alumbramiento activo modificado, 1,54 $\pm 0,53$, si lo comparamos con el número de tollas utilizadas en el alumbramiento activo, $143 \pm 0,54$, con significación estadística $(\mathrm{p}=$ 0,009). Debemos enfatizar que el método de mensurar la cantidad de sangre en la primera hora del posalumbramiento, de acuerdo al número de toallas cambiadas, no es exacto. Se trata de una valoración aproximada que no mide con precisión la cantidad de sangre, por lo que estos resultados hay que valorarlos considerando esta explicación. No se dispuso de una balanza de altísima precisión, para pesar las toallas antes de aplicarlas en los genitales externos maternos y luego con el peso de la sangre recogida.

Durante la investigación no existieron abandonos de embarazadas luego de la aleatorización e ingreso en la investigación, razón por lo que no fue necesario obtener los resultados de acuerdo a la intención a tratar. Considerando las diferencias, los resultados de la investigación sobre el tiempo de duración del alumbramiento y la pérdida de sangre son mejores que los demostrados por el metaanálisis de Prendiville y col. (2000) de la base de datos Cochrane. Este metaanálisis demostró que el alumbramiento dirigido (equivalente al alumbramiento activo de nuestra investigación), que utilizó ocitócicos, encontró para la duración del alumbramiento un tiempo medio de 9,77 minutos. En el presente estudio las medias de duración del alumbramiento fueron menores: 4,35 $\pm 1,36$ minutos para el alumbramiento activo modificado y 4,22 $\pm 1,60$ minutos para el alumbramiento activo, con un valor de $\mathrm{p}$ de 0,297 . Además, el metaanálisis confirmó que el alumbramiento dirigido estuvo asociado con un incremento materno de náusea y vómito, con un RR de 1,83 y un IC95\% de 1,51 a 2,23, probablemente por el uso de ergometrina, hallazgo no presente en la investigación, ya que se utilizó solamente ocitocina en los dos tipos de atención del alumbramiento y no ergometrina. Por lo expuesto los resultados superan los hallazgos del metaanálisis mencionado ya que se consiguió, además, disminuir la media de la pérdida de sangre a 193,01 \pm 66,97 cc con la atención del alumbramiento activo modificado y a 182,97 $\pm 54,62$ cc con la atención del alumbramiento activo $(\mathrm{p}=0,045)$. 
A pesar de los resultados beneficiosos del alumbramiento activo modificado y del alumbramiento activo, no se comprobó que los resultados de la atención del alumbramiento activo modificado sean superiores a los de la atención del alumbramiento activo. La interpretación de los resultados permite concluir que los resultados de los dos tipos de atención del alumbramiento son estadísticamente iguales, ya que la mayoría de las variables, con excepción de la pérdida de sangre, se comportaron de igual manera como respuesta a la aplicación de los dos procedimientos de atención.

Se considera que el tacto vaginal, que comprueba con certeza el desprendimiento placentario, durante la atención del alumbramiento activo modificado, tiene mayor eficacia para diagnosticar el desprendimiento placentario, que los signos indirectos e inciertos de la contracción uterina y del descenso del cordón umbilical, utilizados en el alumbramiento activo, para iniciar las maniobras de extracción placentaria. Si en la atención del alumbramiento activo los dos signos: contracción uterina y/o descenso del cordón umbilical, no son signos reales de desprendimiento placentario y se inicia la tracción del condón umbilical se podría producir un desprendimiento incompleto de la placenta lo que constituye una complicación hemorrágica grave.

\section{CONCLUSIONES}

La investigación demostró que los dos tipos de atención del alumbramiento activo modificado y alumbramiento activo, disminuyeron notoriamente el tiempo de duración del alumbramiento y la pérdida de sangre a niveles tan bajos, que induce a no admitir y aún a rechazar algunas recomendaciones internacionales sobre la atención del alumbramiento. No se comprobó que los resultados de la atención del alumbramiento activo modificado sean mejores que los de la atención del alumbramiento activo, hipótesis de la investigación. Sin embargo, la interpretación de los hallazgos permite concluir que los resultados de los dos tipos de atención del alumbramiento son estadísticamente semejantes, ya que la mayoría de las variables dependientes se comportan de igual manera como respuesta a las variables independientes del alumbramiento activo modificado y del alumbramiento activo.

Una retención de restos placentarios en 300 pacientes atendidas, en la atención del alumbramiento activo modificado y una retención de membranas en el alumbramiento activo en 300 pacientes atendidas, corresponde a las encontradas en estadísticas internacionales. No existió retención total de la placenta en las dos variedades de atención del alumbramiento. Conociendo los resultados beneficiosos obtenidos de las dos formas de atención del alumbramiento, utilizando para ello, el rigor metodológico de un estudio experimental aleatorizado, consideramos que al ser aplicadas las normas de atención de las dos variedades del alumbramiento en los centros de atención obstétrica, contribuirán a reducir en gran magnitud la morbilidad y mortalidad por hemorragia durante el parto, que actualmente constituye la segunda causa de muerte materna en nuestro país y en el mundo.

\section{AGRADECIMIENTO}

Es un deber y obligación agradecer a la Dirección de Investigación de la Universidad de Cuenca (DIUC) y a la Dra. Janneth Ávila, Coordinadora del Centro de Apoyo a la Mujer y la Familia. Al personal docente y de servicio médico del Centro Obstétrico del Hospital Vicente Corral Moscoso y de la Facultad de Ciencias Médicas, a los estudiantes ayudantes de investigación (Gabriel Flores Enderica y Ángel Herrera Lema). Muchas gracias a todas las personas que participaron en la fase de investigación y experimento: Dr. Carlos Flores D, Médicos Residentes del Curso de Postgrado de Ginecología y Obstetricia de la Facultad de Ciencias Médicas de la Universidad de Cuenca: Dr. Roberto Ariño Arias, Dra. María Augusta Arias Chuchuca, Dra. Marina Rodríguez Déleg, Dra. Diana Ramírez Cabrera, Dra. Elsa Cuenca Condoy, Dra. Gloria Franco Ruiz, Dra. Eulalia Agudo Carpio, Dr. Omar Guevara Pérez, Dra. Verónica López Alvarado, Dra. Gladys Mendoza Eskola, Dr. Henry 
Vinueza Aguay, Dra. Jéssica Matute Chávez, Dra. María Alexandra Piedra Lazo y Dra. María Elizabeth Heraz Crespo.

\section{BIBLIOGRAFÍA}

Botero, J., Jubiz, A., Henao, G. (Eds.), 2008. Obstetricia y Ginecología: Texto integrado. $8^{\text {a }}$ edición, Editorial Québec World, Bogotá, p. 152-153.

Cárdenas, O., 2003. Estudio comparativo entre el alumbramiento activo y el alumbramiento expectante. Tesis para la Obtención de Maestría en Investigación en Salud. Universidad de Cuenca, Cuenca, Ecuador.

Cárdenas, O., Molina, X., Narváez, J., 2003. Atención del parto en posición vertical materna. Diseño y construcción del equipo médico para la atención del parto en posición vertical materna. $1^{\mathrm{a}}$ edición. Editorial Universidad de Cuenca, Cuenca, Ecuador, p. 58-61, 74.

Carrera, J., Mallabre, D., Bernar, Z., 2006. Protocolos de obstetricia y medicina perinatal. $4^{\text {a }}$ edición. Editorial Masson, España, p. 358-359.

Cifuentes, R. (Ed.), 2009. Ginecología y obstetricia basada en las nuevas evidencias. $2^{\text {a }}$ edición. Editorial Distribuna, Bogotá, p. 23.

Quintero, F., 1999. Estudio comparativo entre la atención del alumbramiento expectante y activo. Tesis de Posgrado de Ginecología y Obstetricia. Universidad de Cuenca, Cuenca, Ecuador.

McDonald, S., Prendiville, W.J., Elbourne, D., 2002. Sintometrina profiláctiva versus oxitocina en el alumbramiento. $5^{\mathrm{a}}$ edición. Biblioteca de Salud Reproductiva de la OMS, CD-ROM Versión 1.0.

Ministerio de Salud Pública, 2008. Norma y protocolo materno. $1^{\text {a }}$ edición, MSP, Quito, Ecuador, p. 46-47.

Organización Mundial de la Salud (OMS), 2001. Manejo de las complicaciones del embarazo y el parto. $1^{a}$ edición. WHO/RHR/00.7, $438 \mathrm{pp}$.

Pérez Sánchez, A., Donoso Siña, E., 2011. Obstetricia. $4^{a}$ edición. Editorial Mediterráneo, Santiago de Chile, p. 360-362.

Prendiville, W., Elbourne, D., McDonald, S., 2000. Active versus expectant management in the third stage of labor. Meta-analyses Cochrane Review. The Cochrane Database Syst. Rev., Issue 3, CD000007, Oxford, UK.

Rogers, J., Wood, J., McCandlish, R., Ayers, S., Truesdale, A., Elbourne, D., 1998. Active versus expectant management of third stage of labour: the Hinchingbrooke randomized controlled trial. Lancet, 351(9104), 693-699.

Schwarcz, R., Fescina, R., Duverges, C., 2005. Obstetricia. 6ª edición. Editorial El Ateneo, Buenos Aires, Argentina, p. 491-494. 